\title{
MONOIDAL CLOSED STRUCTURES ON CATEGORIES WITH CONSTANT MAPS
}

\author{
ALESSANDRO LOGAR and FABIO ROSSI
}

(Received 23 November 1983)

Communicated by R. H. Street

\begin{abstract}
The purpose of this paper is to study the so-called canonical monoidal closed structures on concrete categories with constant maps. First of all we give an example of a category of this kind where there exists a non canonical monoidal closed structure. Later, we give a technique to construct a class of suitable full subcategories of the category of $T_{0}$-spaces, such that all monoidal closed structures on them are canonical. Finally we show that "almost all" useful categories of topological compact spaces admit no monoidal closed structures whatsoever.
\end{abstract}

1980 Mathematics subject classification (Amer. Math. Soc.): 18 D 15, 18 B 30, 54 B 30.

\section{Introduction}

Monoidal closed structures (symmetric or not) on ( $E, M)$-topological categories have been recently examined by many people.

In this direction, Greve [3], [4], [5], generalizing a Booth and Tillotson's theorem [1] has shown that in many topological categories (topological spaces, uniform spaces, merotopic spaces, etc.) and also in suitable initially structured categories $\left(T_{0^{-}}, T_{1^{-}}, T_{2^{-}}\right.$-spaces) there exists a proper class of different (non symmetric) monoidal closed structures.

Porst and Wischnewsky [10], [11], and independently Činčura [2] have discovered a general technique which allows the construction of a symmetric monoidal closed structure on every initially structured category.

This work was partially supported by the Italian CNR.

(C) 1985 Australian Mathematical Society 0263-6115/85\$A2.00+0.00 
All the (non trivial) examples which can be constructed with the above methods show that the forgetful functor $\mathscr{U}$ over Set is strict monoidal, hence the tensor product of two objects $X, Y$ is, roughly speaking, a suitable structure on $\mathscr{U} X \times \mathscr{U} Y$ and the natural isomorphisms have underlying canonical bijections. Let us call canonical every monoidal closed structure like this.

Enlarging a recent (unpublished?) Niederle's result (compare [2], Theorem 1.11) one can see that, for a suitable concrete category with constant maps and surjective epimorphisms, this is a general situation; namely every monoidal closed structure on this category is canonical (compare Theorem 1.4). A large class of categories satisfies the above theorem; for instance all topological categories. Hence the assumptions of constant maps and surjective epimorphisms seem to be crucial.

In Section 2 of this paper we show however, that this impression is false; we give in fact an example of a non trivial category of this kind, where there exists a non canonical monoidal closed structure.

On the other hand from a well known Isbell's result [7] it comes that also on the category of $T_{0}$-spaces (and continuous maps), all monoidal closed structures are canonical although not all epimorphisms are surjective, therefore previous Theorem 1.4 does not apply.

It is now natural to wonder if there exists any other initially structured category in which epimorphisms are in general not surjective, and where all monoidal closed structures are canonical.

In Section 3 we give a technique to construct a class of suitable full subcategories of the category of $T_{0}$-spaces, which affirmatively answers to the above question as is shown in the examples of Section 4.

Finally, an extremal situation about categories with constant maps is examined in Section 5, where it is shown that "almost all" useful categories of topological compact spaces admit no monoidal closed structures whatsoever.

The authors are indebted to Max Kelly and Fabio Zanolin for many helpful conversations on the topics covered by this paper.

\section{Preliminaries}

The aim of this section is to recall some results about monoidal closed structures on concrete categories with constant maps.

These results seem to be known in the particular case where the monoidal closed structure is also symmetric (compare [2], Theorem 1.11). The proofs, however, seem to be unpublished and therefore we will give here them for the general case. 
Let $(\mathscr{A}, \mathscr{U}: \mathscr{A} \rightarrow$ Set) be a concrete category, that is, the functor $\mathscr{U}$ is faithful and transportable. We say that $(\mathscr{A}, \mathscr{U})$ admits constant maps iff every constant morphism $\mathscr{U} A \rightarrow \mathscr{U} B$ underlies some $\mathscr{A}$-morphism $A \rightarrow B$, and we denote by $\operatorname{Cost}(X, Y)$ the set of constant functions $X \rightarrow Y$ for any $X, Y \in$ Set.

The symbol 1 shall be used for a terminal object of Set.

PROPOSITION 1.1. A category $\mathscr{A}$ is a concrete $(\mathscr{A}, \mathscr{U})$ with constant maps and with an object $I$ such that $\mathscr{U} I=1$ iff $I$ is terminal and generator in $\mathscr{A}$.

Proof. Since $\operatorname{Cost}(\mathscr{U} A, \mathscr{U} I) \subseteq \mathscr{A}(A, I) \subseteq \operatorname{Set}(\mathscr{U} A, \mathscr{U} I)$ and $\mathscr{U} I=1$, then $\mathscr{A}(A, I)=1$, so $I$ is terminal. Since $\operatorname{Cost}(\mathscr{U} I, \mathscr{U} A) \cong \mathscr{U} A \subseteq \mathscr{A}(I, A) \subseteq$ $\operatorname{Set}(\mathscr{U} I, \mathscr{U} A) \cong \mathscr{U} A$, then $\mathscr{U}(-) \cong \mathscr{A}(I,-)$ and $I$ is generator.

Conversely, let $I$ be terminal and generator in $\mathscr{A}$; then $(\mathscr{A}, \mathscr{A}(I,-): \mathscr{A} \rightarrow$ Set) is a concrete category with $\mathscr{A}(I, I)=1$. If

$$
k: \mathscr{U} A=\mathscr{A}(I, A) \rightarrow \mathscr{U} B=\mathscr{A}(I, B)
$$

is a constant map, then $k$ can be written as a composite

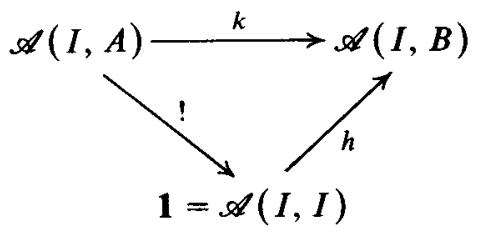

so $k=\mathscr{A}(I, h(1) !)=\mathscr{U}(h(1) !)$, where !: $A \rightarrow I$.

Let now $\mathscr{A}$ be a category with the following properties:

(a) $\mathscr{A}$ is a concrete $(\mathscr{A}, \mathscr{U})$;

(b) $(\mathscr{A}, \mathscr{U})$ admits constant maps;

(c) there exists an $\mathscr{A}$-object $\bar{A}$ with $\operatorname{card}(\mathscr{U} \bar{A}) \geqslant 2$ (i.e. $\mathscr{A}$ is not a preorder).

Proposition 1.2. Every monoidal closed structure $(-\square-, I, r, l, a,[-,-])$ on $\mathscr{A}$ satisfies (up to natural isomorphisms) the following:

(1) $\mathscr{U I}=1 ; I$ is terminal and generator in $\mathscr{A}$;

$$
\mathscr{U}(-)=\mathscr{A}(I,-) \text {; }
$$

(2) $\mathscr{U}[B, C]=\mathscr{A}(B, C)$ for any $B, C \in \mathscr{A}$;

(3) $\mathscr{U} A \times \mathscr{U} B \subseteq \mathscr{U}(A \square B)$, where the inclusion $i$ is natural in $A$ and $B$;

(4) The inclusion $i$ is $\mathscr{U}$-epic (i.e. $(\mathscr{U} h) i=(\mathscr{U} k) i \Rightarrow h=k$ for any $h, k$ : $A \square B \rightarrow C$ );

(5) If $\pi: \mathscr{A}(A \square B, C) \cong \mathscr{A}(A,[B, C])$ is the adjunction, then

$$
[(\mathscr{U} \pi(f))(x)](y)=(\mathscr{U} f)(x, y)
$$

and

$$
\left(\mathscr{U} \pi^{-1}(g)\right)(x, y)=((\mathscr{U} g)(x))(y),
$$


for any $f: A \square B \rightarrow C, g: A \rightarrow[B, C],(x, y) \in \mathscr{U} A \times \mathscr{U} B$

$$
\begin{gathered}
\mathscr{U} I \times \mathscr{U} A=\mathscr{U}(I \square A) ; \quad \mathscr{U} A \times \mathscr{U} I=\mathscr{U}(A \square I) ; \\
(\mathscr{U} l)(*, x)=x ; \quad(\mathscr{U} r)(x, *)=x ; \\
((\mathscr{U} a) i)((x, y), z)=(x,(y, z)) .
\end{gathered}
$$

Proof. $\mathscr{A}(I, I)$ is a commutative monoid, so card( $\mathscr{U} I) \leqslant 1$. If $\mathscr{U} I=\varnothing$ then $\operatorname{card}(\mathscr{A}(A, B)) \leqslant 1$ for any $A, B \in \mathscr{A}$ because $\mathscr{A}(A, B) \cong \mathscr{A}(I \square A, B) \cong$ $\mathscr{A}(I,[A, B])$ and $\operatorname{card}(\mathscr{A}(I,[A, B])) \leqslant 1$; since $\mathscr{A}$ is not a preorder, $\mathscr{U} I$ must be a singleton. Then, by $1.1, I$ is terminal and generator in $\mathscr{A}$ and $\mathscr{U}(-) \cong \mathscr{A}(I,-)$; moreover $\mathscr{U}[B, C] \cong \mathscr{A}(I,[B, C]) \cong \mathscr{A}(I \square B, C) \cong \mathscr{A}(B, C)$ so (up to obvious identifications), (1) and (2) follow.

The functor $\mathscr{U}$ becomes then a monoidal one with natural transformation

$$
i_{A, B}: \mathscr{U} A \times \mathscr{U} B \rightarrow \mathscr{U}(A \square B)
$$

defined by $i_{A, B}(x, y)=(x \square y) l^{-1}, x: I \rightarrow A, y: I \rightarrow B$. If $t$ is defined by the following diagram

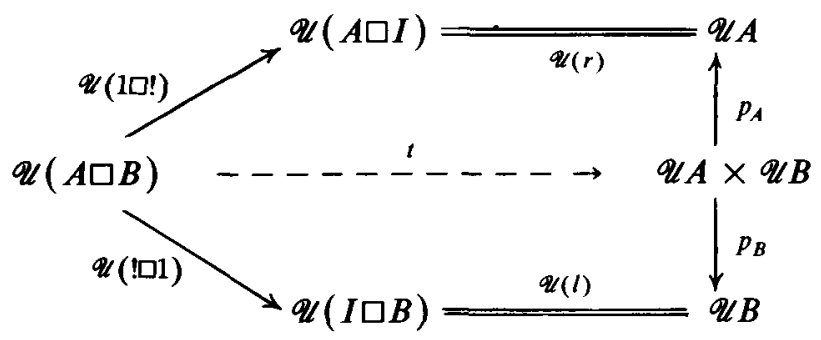

it is easy to see that $t i=1_{q A \times q B}$, then (3) holds.

$$
\begin{aligned}
& (\mathscr{U} h) i=(\mathscr{U} k) i \text { iff } h(x \square y)=k(x \square y) \text { if and only if } \\
& {[y, 1] \pi(h) x=[y, 1] \pi(k) x,}
\end{aligned}
$$

for any $x: I \rightarrow A, y: I \rightarrow B$. Applying $\mathscr{U}$, we obtain

$$
\begin{aligned}
& (\mathscr{U} \pi(h))(x) y=(\mathscr{U} \pi(k))(x) y, \text { for any } x \in \mathscr{U} A, y: I \rightarrow B ; \\
& \text { then } \mathscr{U} \pi(k)=\mathscr{U} \pi(h)
\end{aligned}
$$

and so $h=k$ and (4) holds.

If $f: A \square B \rightarrow C$ is an $\mathscr{A}$-morphism, then $(\mathscr{U} f)(x,-)$ and $(\mathscr{U} f)(-, y)$ underlie the $\mathscr{A}$-morphisms $f(x \square 1) l^{-1}$ and $f(1 \square y) r^{-1}$, for any $x: I \rightarrow A, y: I \rightarrow B$, respectively.

Let us consider now the function $\mathscr{U} \pi(f): \mathscr{U} A \rightarrow \mathscr{U}[B, C]$ evaluated to an arbitrary $x: I \rightarrow A$. We have

$$
(\mathscr{Q} \pi(f))(x)=\pi^{-1}(\pi(f) x) l^{-1}=f(x \square 1) l^{-1},
$$

then (5) holds.

(6) is trivial. 
Definition 1.3. Let $\mathscr{A}$ be a concrete category. A monoidal closed structure on $\mathscr{A}$ which verifies the conditions (1), (2), (4), (5), (6) of 1.2 and, instead of (3), the stronger:

(3') $\mathscr{U} A \times \mathscr{U} B=\mathscr{U}(A \square B)$ for all $A, B \in \mathscr{A}$,

is called canonical.

Of course, for every canonical monoidal closed structure on $\mathscr{A}$, the natural isomorphisms $a, r, l$ and the adjunction $\pi$ have the obvious underlying bijections.

THEOREM 1.4. If $\mathscr{A}$ is a category that verifies the previous conditions (a), (b), (c) and also:

(d) for every $X \subseteq \mathscr{Q} A$ there exists a morphism $\dot{J}: B \rightarrow A$ such that $\mathscr{U} B=X$ and $\mathscr{Q} \dot{J}$ is the inclusion;

(e) $\mathscr{U}$ preserves epimorphisms, then all monoidal closed structures on $\mathscr{A}$ are canonical (up to natural isomoprhisms).

REMARK 1.5. Many categories satisfy the Theorem 1.4; for instance every topological category in Herrlich's sense (compare [6]) or, more generally, every initially structured category in Nel-Wyler's sense (compare [9], [12]) such that the forgetful functor preserves epimorphisms (like for example, $T_{1}$-spaces, closure spaces, partially ordered sets, generalized topological space in Čech's sense, and so on), (compare also [2]).

\section{A counterexample}

Let $\mathscr{S}$ be the category of semilattices and functions which preserve binary suprema, and let $\mathscr{U}: \mathscr{S} \rightarrow$ Set be the obvious forgetful functor. We denote with $\mathscr{S}_{\text {fin }}$ the full subcategory of $\mathscr{S}$ whose objects are the finite semilattices.

Proposition 2.1. The category $\mathscr{S}_{\text {fin }}$ verifies conditions (a), (b), (c), (e) of Section 1 , but there exists a non canonical monoidal closed structure on it.

Proof. first of all we consider the category $\mathscr{S}$. Observe that an object of $\mathscr{S}$ is a set $X$ with a binary composition law $\vee: X \times X \rightarrow X$ which is idempotent, associative and commutative; so we could see $\mathscr{S}$ as a category of $(\Omega, E)$-algebras (or, equivalently, as an Eilenberg-Moore category $\operatorname{Set}^{T}$ for a suitable monad $\mathbf{T}$ over Set). (Compare [8].)

Let $X, Y, Z$ be semilattices. We will call bimorphism every function $f$ : $X \times Y \rightarrow Z$ such that $f(x,-): Y \rightarrow Z$ and $f(-, y): X \rightarrow Z$ are $\mathscr{S}$-morphisms for every $x \in X, y \in Y$. ( $\mathscr{U}$ is omitted.) 
Let $F(X \times Y)$ be the free semilattice on the set $X \times Y$, and let $X \otimes Y$ be the quotient $F(X \times Y) / \mathbf{R}$ where $\mathbf{R}$ is the smallest congruence relation on $F(X \times Y)$ such that the function $\otimes: X \times Y \stackrel{i}{\rightarrow} F(X \times Y) \stackrel{\rho}{\rightarrow} F(X \times Y) / \mathbf{R}$ is a bimorphism. It is easy to see that $\otimes$ satisfies the usual universal property with respect to all bimorphisms. Giving the obvious semilattice structure on $\mathscr{P}(Y, Z)$ we obtain a symmetric monoidal closed structure on $\mathscr{S}$.

Observe that one can find the above monoidal closed structure on $\mathscr{S}$, using the general Porst and Wischnewsky's technique as in [10], Section 4; in fact $\mathscr{S}$ is an Eilenberg-Moore category, and $\mathscr{U}: \mathscr{S} \rightarrow$ Set is semitopological functional functor.

It is easy to see that the above structure on $\mathscr{S}_{\text {can }}$ be restricted on $\mathscr{S}_{\text {fin }}$. Let now $X \in \mathscr{S}_{\text {fin }}$ be the two element semilattice $\{0,1\}$ with $0<1$, and let $a=(0,0)$, $b=(0,1), c=(1,0), d=(1,1)$ the four elements of $X \times X$. It is easy to see that $F(X \times X)=\{a, b, c, d, a b, a c, a d, b c, b d, c d, a b c, a b d, a c d, b c d, a b c d\}(\vee$ is omitted), and that $\mathbf{R}$ is the following partition: $\{a\},\{b, a b\},\{c, a c\}$, $\{d, a d, b d, c d, a b d, a c d, b c d, a b c d\},\{b c, a b c\}$.

This shows that $X \otimes X$ has five elements, and therefore the considered structure on $\mathscr{S}_{\text {fin }}$ (and on $\mathscr{S}$ ) cannot be canonical.

Moreover $\mathscr{S}_{\text {fin }}$ satisfies conditions (a), (b), (c), (e) of Section 1: (a), (b), (c) are trivial; condition (e) as follows: let $f: A \rightarrow B$ be a non surjective morphism. Because $B$ is finite, there exists $b_{0} \in B \backslash f(A)$ such that if $b_{1}, b_{2} \in B$ and $b_{1}<b_{0}$, $b_{2}<b_{0}$ then $b_{1} \vee b_{2}<b_{0}$. If we call $B_{1}=\left\{b \in B: b \leqslant b_{0}\right\}, B_{2}=\{b \in B$ : $\left.b<b_{0}\right\}$ and again $X$ the two elements semilattice $\{0,1\}(0<1)$, the following two applications $u, v: B \rightarrow X$

$$
u b=\left\{\begin{array}{ll}
0 & \text { if } b \in B_{1}, \\
1 & \text { if } b \in B \backslash B_{1},
\end{array} \quad v b= \begin{cases}0 & \text { if } b \in B_{2}, \\
1 & \text { if } b \in B \backslash B_{2},\end{cases}\right.
$$

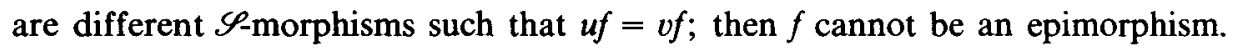
This concludes the proof.

\section{Subcategories of $\mathscr{T}_{0}$ with canonical monoidal closed structures}

Let $\mathscr{T}_{0}$ be the category of $T_{0}$-spaces and continuous maps. We will use "subcategory" to mean "full replete subcategory".

In this section we will give a technique to construct coreflective subcategories of $\mathscr{T}_{0}$, where all monoidal closed structures are canonical, but where Theorem 1.4 is not applicable because not every epimorphism is surjective.

We will use Nel's results [9] about initially structured categories.

First of all we recall the following lemma due to Isbell (compare [7], 1.1). 
LEMMA 3.1. Let us consider the following diagram in $\mathscr{T}_{0}$ :

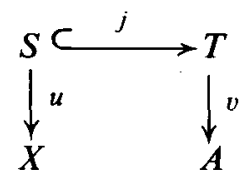

where $X$ is a Hausdorff space, $A, T \in \mathscr{T}_{0}, S$ is a subspace of $T, j$ is the inclusion and $u, v$ are continuous maps. If $j$ is a $\mathscr{T}_{0}$-epimorphism, and the fibers $u^{-1}(h)$ are all mapped homeomorphically by $v$ upon $A$, then $S=T$.

From this it follows:

LEMMA 3.2. Let $\mathscr{A}$ be a coreflective subcategory of $\mathscr{T}_{0}$, and (-口-, I, $\left.r, l, a,[-,-]\right)$ be a monoidal closed structure on $\mathscr{A}$.

For every Hausdorff space $H$ in $\mathscr{A}$ and for every $A \in \mathscr{A}$ we have (up to isomorphisms)

$$
\mathscr{U} H \times \mathscr{U} A=\mathscr{U}(H \square A),
$$

where $\mathscr{U}: \mathscr{A} \rightarrow$ Set is the evident forgetful functor.

Proof. If $\mathscr{A}=\{\varnothing\}, 3.2$ is obvious. Otherwise, $\mathscr{A}$ is non trivial in Nel's sense and so it is initially structured (compare [9], Theorem 1.13); moreover $\mathscr{A}$ verifies Proposition 1.2.

The inclusion $i: \mathscr{U} X \times \mathscr{U} A \hookrightarrow \mathscr{U}(X \square A)$ is a mono-source in Set (for every $X, A \in \mathscr{A})$; so we can give a topology $X \times{ }_{\tau} A$ on $\mathscr{U} X \times \mathscr{U} A$ such that $i$ becomes $\mathscr{U}$-initial mono-source in $\mathscr{A}$. From $1.2,4$ ) it follows that $i$ is an epimorphism in $\mathscr{A}$ and hence also in $\mathscr{T}_{0}$.

Let now $X \times{ }_{s} A$ be the subset $\mathscr{U} X \times \mathscr{U} A$ of $X \square A$, with the induced topology. Because $X \times{ }_{\tau} A$ is finer than $X \times{ }_{s} A$, it follows that the inclusion $j: X \times{ }_{s} A \hookrightarrow$ $X \square A$ is a $\mathscr{T}_{0}$-epimorphism.

Let $\bar{t}$ be the morphism defined by

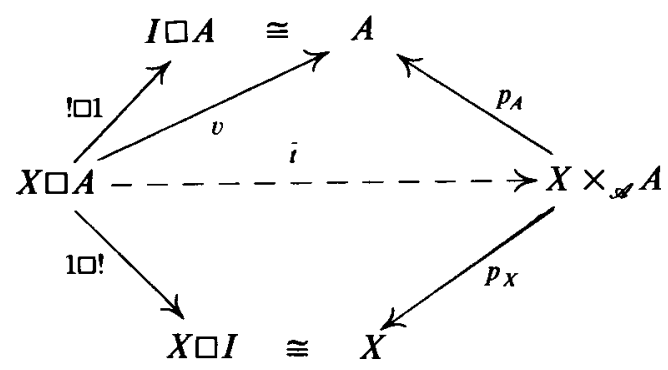

where $X \times \mathscr{A} A$ is the cartesian product in $\mathscr{A}$ (compare [9] Theorem 1.1), and ! are the unique morphisms. The functor $\mathscr{U}: \mathscr{A} \rightarrow$ Set preserves limits, so from 1.2 we 
have $\mathscr{U}(\overline{t j})=1_{\mathscr{U} \times \mathscr{U}_{A}}$, then in the following commutative diagram

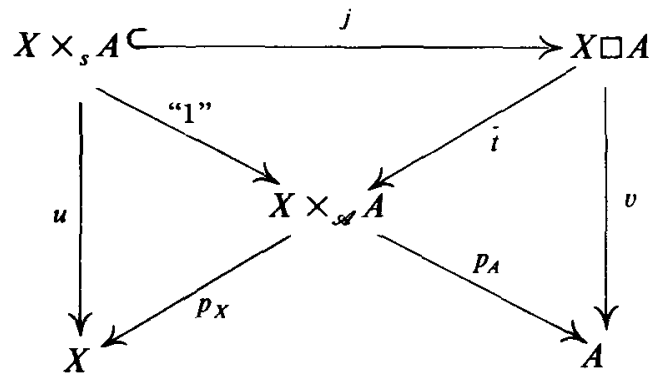

$u$ and $v j$ have as underlying maps just the usual projections.

For every $x \in X,\left.v\right|_{u^{-1}(x)}: u^{-1}(x) \rightarrow A$ is bijective and continuous, and its inverse is also continuous because it is essentially the map $A \cong I \square A \stackrel{x \square 1}{\longrightarrow} X \square A$.

If in particular $X$ is a Hausdorff space, it follows from 3.1 that $X \times{ }_{s} A=X \square A$. This concludes the proof.

Let now $\mathscr{B}$ be a non trivial class of Hausdorff spaces and let $\mathscr{A}=$ hull $_{\mathscr{T}_{0}}(\mathscr{B})$ be the coreflective hull of $\mathscr{B}$ in $\mathscr{T}_{0}$.

THEOREM 3.3. Every monoidal closed structure on $\mathscr{A}$ is canonical (up to natural isomorphisms).

Proof. If $(-\square-, I, r, l, a,[-,-])$ is a monoidal closed structure on $\mathscr{A}$ the only thing to prove is that $\mathscr{U} Y \times \mathscr{U} Z=\mathscr{U}(Y \square Z)$ for every $Y, Z \in \mathscr{A}$, because $\mathscr{A}$ is initially structured.

From [9], 1.12, it follows that there exists a family $\left\{B_{i}\right\}_{i \in I}, B_{i} \in \mathscr{B}$ such that $Y$ is a quotient $p$ (in $\mathscr{T}_{0}$ or equivalently in $\mathscr{A}$ ) of the coproduct $\bigsqcup_{i \in I} B_{i}$.

In the following commutative diagram (compare 1.2,(3))

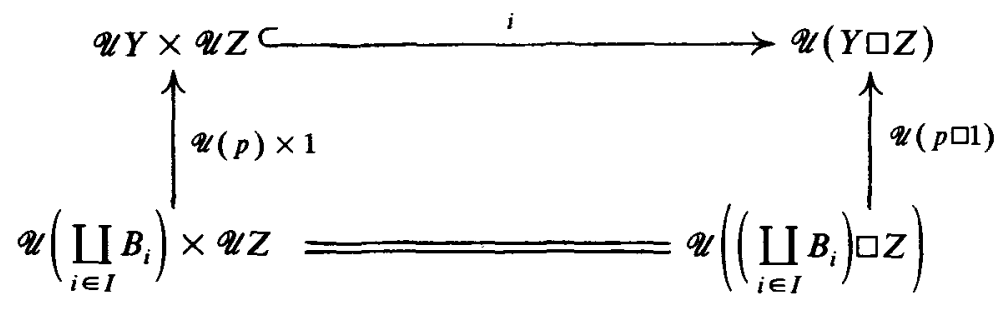

(the equality follows from 3.2 because $\amalg_{i \in I} B_{i}$ is a Hausdorff space in $\mathscr{A}$ ), $p \square 1$ is again a quotient in $\mathscr{A}$ because $-\square Z$ preserves colimits and so $\mathscr{U}(p \square 1)$ is a surjective map (compare [9], 1.4, 1.6). Therefore $i$ is surjective and the thesis follows. 


\section{Examples}

(a) Take $\mathscr{B}$ the class of all uniform ultraspaces defined on all (infinite) cardinals. Then $\mathscr{A}$ is $\mathscr{T}_{0}$, and so we refind that in this category all monoidal closed structures are canonical.

(b) Take $\mathscr{B}=\left\{N_{\infty}\right\}$, the one point compactification of the natural numbers with the discrete topology. Then $\mathscr{A}$ is exactly the category of $T_{0}$-sequential spaces.

(c) More generally, let $\alpha$ be an (infinite) regular cardinal and let $\mathscr{F}_{\alpha}$ be the genearlized Fréchet filter on $\alpha\left(A \in \mathscr{F}_{\alpha}\right.$ if and only if $\left.\operatorname{card}(\alpha \backslash A)<\alpha\right)$. Denote with $C(\alpha)$ the corresponding filter space defined on $\alpha \cup\{\infty\}$. If we take $\mathscr{B}=\{C(\alpha)\}$, then $\mathscr{A}$ is now the category of $T_{0}-\alpha$-sequential spaces.

(d) Take $\mathscr{B}=\{H: H$ is compact Hausdorff space $\}$. Then $\mathscr{A}$ is the category of $T_{0}$-compactly generated spaces.

RemarK 4.1. In all the above examples epimorphisms are not, in general, surjective. For instance, take $X$ the set $N \cup\{\infty\}(N=$ natural numbers) with the following topology:

$$
A \subseteq X \text { is open iff }\left\{\begin{array}{l}
A=\varnothing \\
A=A^{\prime} \cup\{\infty\}, A^{\prime} \subseteq N, N \backslash A^{\prime} \text { finite }
\end{array}\right.
$$

If we take the subspace $X^{\prime}=X \backslash\{\infty\}$ it is easy to see that the inclusion map $i$ : $X^{\prime} \hookrightarrow X$ is a $\mathscr{T}_{0}$-epimorphism because $i^{-1}$ is an injection on open sets. Moreover $X$ and $X^{\prime}$ are sequential spaces, so they give an example of non surjective epimorphism in cases (b), (d) and clearly in (a).

A straightforward generalization gives examples also for case (c).

\section{A negative result}

The goal of this section is to show that in "almost all" useful categories of compact spaces do not exist any monoidal closed structure.

We denote with $-\otimes-:$ Top $\times$ Top $\rightarrow$ Top and $[-,-]_{p c}$ : Top ${ }^{\text {op }} \times$ Top $\rightarrow$ Top the separate continuity tensor product and pointwise convergence internal hom, respectively.

Let $\mathscr{C}$ be a full replete subcategory of Top that contains at least an object with cardinality greater than one and let $(-\square-, I, r, l, a,[-,-])$ be a monoidal closed structure on $\mathscr{C}$. 
From 1.2 we have the natural inclusion: $i: \mathscr{U} X \times \mathscr{U} Y \rightarrow \mathscr{U}(X \square Y)$ (for every $X, Y \in \mathscr{C} ; \mathscr{U}: \mathscr{C} \rightarrow$ Set the obvious forgetful functor) which is separately continuous and so can be lifted to a map $s_{X Y}: X \otimes Y \rightarrow X \square Y$. Clearly $s_{X Y}$ results a natural transformation $s:-\otimes-\rightarrow-\square-: \mathscr{C} \times \mathscr{C} \rightarrow$ Top. Because $\mathscr{C}$ is full in Top, it is easy to see that $s$ defines a natural transformation $\tau_{Y Z}:[Y, Z] \rightarrow[Y, Z]_{p c}$ for every $Y, Z \in \mathscr{C}$, such that the following diagram

$$
\begin{array}{ccc}
\operatorname{Top}(X \otimes Y, Z) & \cong & \operatorname{Top}\left(X,[Y, Z]_{p c}\right) \\
\operatorname{Top}(s, 1) \uparrow & & \uparrow \operatorname{Top}(1, \tau) \\
\operatorname{Top}(X \square Y, Z) & \cong & \operatorname{Top}(X,[Y, Z])
\end{array}
$$

is commutative for every $X, Y, Z \in \mathscr{C}$.

If in particular, we take in the above diagram $X=I=\{*\}$, we find from 1.2, (6) that $s_{I Y}$ is the identity, and so the underlying map of $\tau_{Y Z}$ is the identity.

Let now $\mathscr{C}$ be any class of compact spaces which contains at least a Hausdorff space $X$ with the property that there exist two distinct points $p, q$ that are joined by a path in $X$ (i.e. $X$ is a space not totally pathwise disconnected).

If we think $\mathscr{C}$ as a full (replete) subcategory of Top, we will prove

THEOREM 5.1. There is not any monoidal closed structure on $\mathscr{C}$.

Before proving the above theorem we recall the following:

LeMma 5.2. Let $X$ be a compact Hausdorff not totally pathwise disconnected space. Then $[X, X]_{p c}$ is not compact.

Proof. Let $X^{X}$ be the set of all functions from $X$ to $X$ with the usual product topology. $X^{X}$ is a Hausdorff space and so the only thing to prove is that its subspace $[X, X]_{p c}$ is not closed.

Let $I_{1}=[0,1]$ be the closed unit interval and let $f: I_{1} \rightarrow X$ be a continuous map such that $f(0)=p, f(1)=q, p \neq q$. The function $g: X \rightarrow X$ defined by

$$
g x= \begin{cases}p & \text { if } x=p, \\ q & \text { otherwise }\end{cases}
$$

is not continuous because $\{p\}$ is not open and $X$ is Hausdorff. Let

$$
\begin{aligned}
& W=W\left(\left\{x_{1}\right\}, U_{1}\right) \cap \cdots \cap W\left(\left\{x_{n}\right\}, U_{n}\right) \\
& \left(U_{1}, \ldots, U_{n} \text { open in } X, W\left(\left\{x_{i}\right\}, U_{i}\right)=\left\{\Phi \in X^{X}: \Phi\left(x_{i}\right) \in U_{i}\right\}\right)
\end{aligned}
$$

be an open basic neighborhood of $g$. Let $S=\left\{x_{1}, \ldots, x_{n}\right\}$. Take $V$ an open neighborhood of $p$ which does not contain the points of $S \backslash\{p\}$ (if $S \backslash\{p\}=\varnothing$, 
take $V$ any open neighborhood of $p$, different from $X$ ). $X$ is completely regular, so there exists a Urysohn function $h$ for $X \backslash V$ and $\{p\}$ such that $h p=0$ and $h(X \backslash V)=1$. The function $f h$ is continuous and $f h \in W$; so $g$ is in the closure of $[X, X]_{p c}$ in $X^{X}$.

Proof of 5.1. Suppose that $(-\square-, I, r, l, a,[-,-])$ is a monoidal closed structure on $\mathscr{C}$. Take $X$ like in the hypothesis of 5.2. Then $\tau_{X X}:[X, X] \rightarrow[X, X]_{p c}$ is surjective as we have seen, and this is a contradiction.

\section{References}

[1] P. Booth and J. Tillotson, 'Monoidal closed, cartesian closed and convenient categories of topological spaces', Pacific J. Math. 88 (1980), 35-53.

[2] J. Čnčura, 'On a tensor product in initially structured categories', Math. Slovaca 29 (1979), $245-255$.

[3] G. Greve, 'How many monoidal closed structures are there in Top?', Arch Math. (Basel) 34 (1980), 538-539.

[4] G. Greve, Rigid spaces and monoidal closedness, 105-111, (Lecture Notes in Math., 915, 1982).

[5] G. Greve, General construction of monoidal closed structures in topological, uniform and nearness spaces, 100-114, (Lecture Notes in Math., 962, 1982).

[6] H. Herrlich, Categorical topology 1971-1981, General topology and its relations to modern analysis and algebra V, Proc. 5th Prague Topol. Symp. 1981 (1983), 279-383.

[7] J. Isbell, 'Function spaces and adjoints', Math. Scand. 36 (1975), 317-339.

[8] E. Manes, Algebraic theories, Springer-Verlag, New York-Heidelberg-Berlin, (1976).

[9] L. D. Nel, 'Initially structured categories and cartesian closedness', Canad. J. Math. 27 (1975), 1361-1377.

[10] H. Porst and M. Wischnewsky, 'Every topological cateogory is convenient for Gel'fand duality', Manuscripta Math. 25 (1978), 169-204.

[11] H. Porst and M. Wischnewsky, Existence and applications of monoidally closed structures in topological categories, 277-292, (Lecture Notes in Math., 719, 1979).

[12] O. Wyler, Function spaces in topological categories, 411-420, (Lecture Notes in Math., 719, 1979).

Istituto di Matematica,

Università Degli Studi di Trieste

Piazzale Europa, 1

34100-Trieste

Italy 